\title{
Sustained Low-Efficiency Daily Diafiltration for Diabetic Nephropathy Patients with Acute Kidney Injury
}

\author{
Xinghua Chen ${ }^{\mathrm{a}} \mathrm{Te}^{\prime}$ an $\mathrm{Ma}^{\mathrm{b}}$ \\ ${ }^{a}$ Division of Nephrology, Renmin Hospital of Wuhan University, Wuhan, and ${ }^{b}$ Division of Nephrology, \\ The First Hospital of Jing Zhou, Yangtze University, Jingzhou, China
}

\section{Key Words}

Acute kidney injury - Diabetic nephropathy - Continuous renal replacement treatment . Sustained low-efficiency daily diafiltration

\begin{abstract}
Objective: To investigate the efficacy, safety and cost of treating patients with acute kidney injury (AKI) and diabetic nephropathy (DN) with continuous renal replacement therapy (CRRT) or sustained low-efficiency daily diafiltration with hemofiltration (SLEDD-f). Subjects and Methods: Medical records of patients with AKI/DN from January 2006 to December 2012 were reviewed. Fifty-five patients who received CRRT and 52 who received SLEDD-f were included in the study. CRRT and SLEDD-f were performed for 20-72 $\mathrm{h}$ per session and 8-10 h per session, respectively. Mortality and renal function recovery rates were evaluated 30 days after the initiation of renal replacement therapy (RRT) and APACHE-II and SOFA scores, anticoagulant dose, inflammatory indices and cost were calculated at baseline and at the end of RRT. Results: Of the 55 patients treated with CRRT, 49 (89.1\%) had a 30-day survival rate and 30 (54.5\%) had a 30-day renal recovery rate. Of the 52 patients with SLEDD-f,
\end{abstract}

these rates were $92.3 \%(n=48)$ and $61.5 \%(n=32)$, respectively. The dosage of low-molecular-weight heparin in the CRRT and SLEDD-f groups was 15,230 $\pm 1,460$ and 6,320 \pm $490 \mathrm{U} /$ day, respectively. The cost of hemopurification and the total cost for patients treated with CRRT was CNY 28,628 $\pm 5,576$ (USD 4,210 \pm 820 ) and CNY 38,828 $\pm 6,324$ (USD $5,710 \pm 930$ ), respectively. These were higher than those for patients treated with SLEDD-f at CNY 13,260 $\pm 1,564$ (USD $1,950 \pm 230$ ) and CNY 19,720 $\pm 2,652$ (USD 2,900 \pm 390 ), respectively. Conclusions: SLEDD-f offered a similar chance of renal recovery and also had further advantages such as a lower heparin dosage, a shorter therapy time and lower hospitalization costs for patients than CRRT. Studies with larger, randomized sample sizes are needed to confirm these findings.

๑ 2014 S. Karger AG, Basel

\section{Introduction}

Diabetic nephropathy (DN) accounts for a large proportion of the potential cost of diabetes to both individuals and society $[1,2]$. In many areas such as cardiovasology, cardiac surgery and endocrinology, it is commonly

Xinghua Chen, $\mathrm{MD}, \mathrm{PhD}$

Division of Nephrology, Renmin Hospital of Wuhan University 238 Jiefang Rd

Wuhan, Hubei 430060 (China)

E-Mail chenxh9905@163.com 
accompanied by acute kidney injury (AKI). AKI concurrent with $\mathrm{DN}(\mathrm{AKI} / \mathrm{DN})$ can be induced by serious infection, hypertension, hypotension, contrast agents, drugs, surgery and renal venous thrombosis and usually leads to multiple organ failure, high mortality and an extensive burden on patients $[3,4]$. AKI is not an isolated event, and it involves distant organ injury including the lungs, heart, liver and brain [4]. The prognosis for patients with $\mathrm{AKI} / \mathrm{DN}$ is poor compared to AKI patients without $\mathrm{DN}$. For critically ill patients, any decrease in renal function could worsen outcome and increase mortality [5] as their condition changes from risk through injury to failure based on the RIFLE (Risk, Injury, Failure, Loss, End Stage renal disease) criteria [6]. The optimal mode of renal replacement therapy (RRT) for such patients is not yet known. Continuous renal replacement therapy (CRRT) is generally used in AKI patients with hemodynamic instability $[7,8]$. However, despite its widespread use, no definitive studies have shown continuous therapy to be superior or even more hemodynamically pleasing than intermittent hemodialysis (IHD). Therefore, it is important to find the optimal mode of RRT for this population.

Since recently, sustained low-efficiency daily dialysis with hemofiltration (SLEDD-f) is increasingly being used in patients with intensive AKI. The SLEDD-f, a hybrid renal replacement technique, differs from conventional hemodialysis mainly in the length of treatment time ( $>8$ vs. $4 \mathrm{~h}$ ) and in blood and dialysate pump velocity (100-200 vs. $200-400 \mathrm{ml} / \mathrm{min}$ and $100-$ 300 vs. $500 \mathrm{ml} / \mathrm{min}$, respectively) [9, 10]. The hybrid renal replacement technique possesses the advantages of conventional hemodialysis and slow continuous therapies and reduces their inherent limitations, such as high costs and logistics and technical complications [9-12].

Recent studies have shown that patients treated with high-volume hemofiltration, daily IHD or SLEDD-f demonstrate lower mortality and an improved solute clearance [13-15]. However, studies on the application of SLEDD-f in patients with AKI/DN are still limited [13, 16]. Until recently, only CRRT was available in our Nephrology Department and medical intensive care unit (ICU). Now SLEDD-f has become an alternative for hemodynamically unstable patients. In this retrospective study, we compared the system coagulation/thrombosis episodes and mortality of patients who were treated only with CRRT and those treated only with SLEDD-f. We also evaluated the cost of CRRT compared to SLEDD-f in $\mathrm{AKI} / \mathrm{DN}$ patients.

\section{Subjects and Methods}

All medical records of AKI/DN patients who received either SLEDD-f or CRRT in the Nephrology Department or the medical ICU of our hospital from January 2006 to December 2012 were reviewed. The study was approved by the IRB. The primary indication for these sustained modalities at our institution is hemodynamic instability as defined by the nephrologists Junzhang Cheng and Hong Jiang. Typically, hemodynamically stable patients receive IHD. In order to choose patients with similar characteristics and to minimize confounding, the following inclusion criteria were used: patients clinically diagnosed with DN (Mogensen criterion [17], stage IV), AKI based on the AKI Network criteria [18], an Acute Physiology and Chronic Health Evaluation II (APACHE-II) score $>10$ [19], a Sepsis-Related Organ Failure Assessment (SOFA) score $>8$ [20] and baseline serum creatinine $(\mathrm{SCr})<5.0 \mathrm{mg} / \mathrm{dl}(442.0 \mu \mathrm{mol} / \mathrm{l})$. The exclusion criteria were concomitant malignant tumor, kidney transplant, surgery due to septic shock or baseline $\mathrm{SCr}>5.0 \mathrm{mg} / \mathrm{dl}$.

During this period, a total of 154 patients received hemopurification treatment. One hundred and seven of them met the inclusion criteria and of these, 55 received CRRT and 52 received SLEDD-f treatment. All treatments were performed using the Gambro dialysis system. The CRRT and SLEDD-f protocols are described below. The ultrafiltration (UF) rate of the dialysis machine did not exceed the replacement fluid rate controlled by an external pump. Utilization of SLEDD-f was decided only if a qualified nurse was available because it requires a high level of nursing acuity. These treatments were monitored by the ICU nursing staff. Because SLEDD-f is labor-intensive, the nurse-to-patient ratio was maintained at 1:1 rather than that the 1:2 required for conventional CRRT. Except for the RRT, all other care was provided by the medical team in the Nephrology Department or ICU.APACHEII scores were calculated at the initiation of dialysis as a predictor of mortality. In addition, clinical progression was monitored using the SOFA score and inflammation indices such as procalcitonin (PCT) and white blood cell count. Anticoagulant dosage and cost were also calculated at the baseline and at the end of RRT.

\section{CRRT Protocol}

CRRT was performed similar to how it is described below except that the replacement fluid was given after filtration at 3,000 $\mathrm{ml} / \mathrm{h}$ and a session lasted $20-72 \mathrm{~h}$. Continuous veno-venous hemofiltration was chosen as the CRRT mode for all the patients. The treatments were performed utilizing the Gambro Prismaflex CRRT machine, and a same high-flux filter (Gambro Prisma M100, AN69 membrane).

\section{SLEDD-f Protocol}

SLEDD-f was performed using the Gambro AK200 Ultra S dialysis machine and the high-flux dialyzer (Gambro Prisma M100, AN69 membrane). Dialysate was produced online with water passing through a portable carbon tank. The SLEDD-f operating parameters at our institution have been largely standardized. Blood flows were set at $150-200 \mathrm{ml} / \mathrm{min}$ and countercurrent dialysate flows were set at $300 \mathrm{ml} / \mathrm{min}$. Replacement fluid was standardized and prefiltrated at $2,000 \mathrm{ml} / \mathrm{h}$. The compositions of the replacement fluid were $140 \mathrm{mEq} / \mathrm{l}$ sodium, $2.15 \mathrm{mEq} / \mathrm{l}$ calcium, $2.0 \mathrm{mEq} / \mathrm{l}$ potassium, $1.0 \mathrm{mEq} / \mathrm{l}$ magnesium, $3 \mathrm{mEq} / \mathrm{l}$ lactate, $32 \mathrm{mEq} / \mathrm{l} \mathrm{bicar-}$ bonate and $110 \mathrm{mg} / \mathrm{dl}$ glucose [15]. Net UF rate was calculated as (replacement fluids + all other fluids in) - (total UF + all other 
Fig. 1. Comparison of single-pass anticoagulant dose, RRT cost and total cost for patients treated with SLEDD-f and CRRT. Differences between the CRRT and SLEDD-f treatment groups were statistically significant. LMWH = Low-molecular-weight heparin.
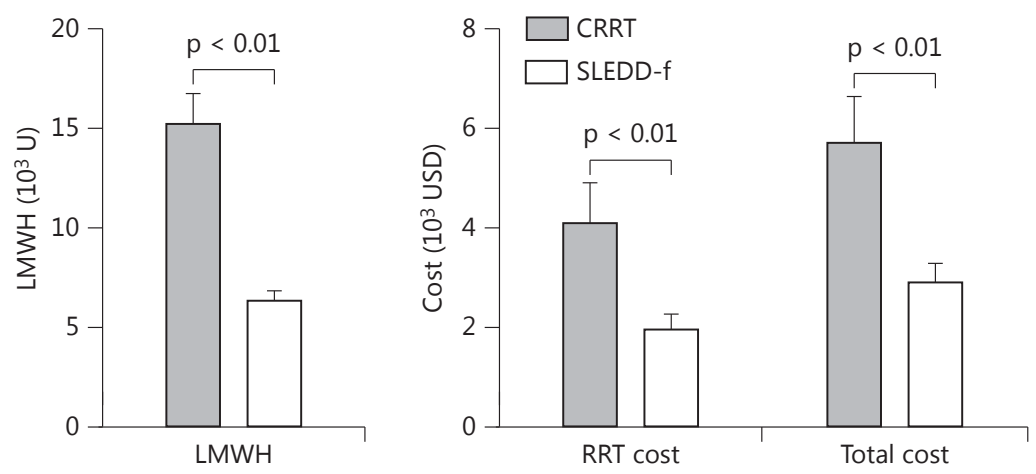

fluids out) and was usually between 0 and $100 \mathrm{ml} / \mathrm{h}$ based on the needs and hemodynamic status of each patient [15]. Serum chemistries were monitored every $6 \mathrm{~h}$. When the serum phosphorus level decreased to $<2.0 \mathrm{mg} / \mathrm{dl}$, sodium phosphate was administered via a peripheral intravenous injection. Time for SLEDD-f was 8-10 $\mathrm{h}$ per session. Low-molecular-weight heparin was administrated at 3,000 U bolus followed by $500 \mathrm{U} / \mathrm{h}$ after $4 \mathrm{~h}$, and the activated partial thromboplastin time was adjusted to 1.5 times of the control value.

CRRT or SLEDD-f was initiated if patients met one of the following criteria [21]: oliguria (urine output $<200 \mathrm{ml} / 12 \mathrm{~h}$ ), anuria (urine output $<50 \mathrm{ml} / 12 \mathrm{~h}$ ), severe acidemia $(\mathrm{pH}<7.1$ ) due to metabolic acidosis, azotemia (urea $>30 \mathrm{mmol} / \mathrm{l})$, hyperkalemia $\left(\mathrm{K}^{+}>6.5\right.$ $\mathrm{mmol} / \mathrm{l}$ or rapid increases in $\mathrm{K}^{+}$), suspected uremic organ involvement (pericarditis/encephalopathy/neuropathy/myopathy), severe dysnatremia $\left(\mathrm{Na}^{+}>160\right.$ or $\left.<115 \mathrm{mmol} / \mathrm{l}\right)$, hyperthermia (core temperature $>39.5^{\circ} \mathrm{C}$ ), clinically significant organ edema (especially in the lungs), drug overdose with dialyzable toxin or coagulopathy requiring large amounts of blood products in a patient at risk of pulmonary edema/acute respiratory distress syndrome.

The criteria for renal recovery included: $\mathrm{SCr}<1.2 \mathrm{mg} / \mathrm{dl}$, urine output $>800 \mathrm{ml} / 24 \mathrm{~h}$ and no need for RRT.

\section{Statistical Analysis}

Normally distributed variables were expressed as mean values \pm SD. Comparison between groups was performed using the SPSS 17.0 software (SPSS Inc., Chicago, Ill., USA), the Student t test, the Mann-Whitney U test (for numerical variables) and the $\chi^{2}$ test (for categorical variables). $\mathrm{p}<0.05$ was considered significant.

\section{Results}

Causes of AKI and Clinical Characteristics of Patients The causes of AKI for patients with DN included infection, biotic factors, heart failure, hypertension and hypotension. No patient underwent noninvasive, assisted ven- tilation such as bilevel positive airway pressure. Patients in both groups had similar causes of AKI and similar clinical characteristics such as age, basal SCr and number of organs experiencing failure, shown in table 1. There were no statistical differences in AKI causes, age or other characteristics.

\section{Patient Outcomes}

The predicted mortality based on the APACHE-II score varied from 20 to $49 \%$ (mean $32.3 \pm 6.9 \%$ ) for patients in the CRRT group and from 18 to $51 \%$ (mean 33.8 $\pm 7.4 \%$ ) for patients in the SLEDD-f group. The CRRT group had a 30 -day survival rate in $89.1 \%(n=49)$ of the patients and a 30 -day renal recovery rate in $54.5 \%(\mathrm{n}=$ 30). For the SLEDD-f group, these rates were $92.3 \%$ ( $n=$ $48)$ and $61.5 \%(n=32)$, respectively. The causes of mortality in patients treated with CRRT were septicemia from pneumococcal infection $(\mathrm{n}=3)$ or cardiogenic shock $(n=1)$, hemorrhage of the digestive $\operatorname{tract}(n=1)$ and myocardial infarction $(n=1)$. The cause of mortality in the SLEDD-f group was septicemia from pneumococcal infection $(n=2)$, cardiogenic shock $(n=1)$ or pancreatitis $(n=1)$. Some patients were off dialysis 30 days after the initiation of SLEDD-f or CRRT.

\section{Clinical Characteristics and Inflammation Index Changes after RRT}

Before treatment, all patients had similar APACHE-II and SOFA scores, renal function, levels of serum blood urea nitrogen and $\mathrm{SCr}$, but after treatment, the APACHEII and SOFA scores and the serum blood urea nitrogen and SCr had decreased in both groups, as shown in table 2 . In addition, inflammation indices such as C-reac- 
Table 1. Causes of AKI/DN and clinical characteristics of patients in the 2 groups

\begin{tabular}{lccc}
\hline & CRRT $(\mathrm{n}=55)$ & SLEDD-f $(\mathrm{n}=52)$ & $\mathrm{p}$ value \\
\hline Causes $^{1}$ & & & \\
$\quad$ Infection & $12(21.8)$ & $11(21.2)$ & 0.933 \\
Antibiotics & $13(23.6)$ & $12(23.1)$ & 0.946 \\
Heart failure & $9(16.4)$ & $8(15.4)$ & 0.890 \\
Hypertension or hypotension & $2(3.6)$ & $2(3.8)$ & 0.954 \\
Two of the above & $9(16.4)$ & $10(19.2)$ & 0.698 \\
Three or more of the above & $10(18.2)$ & $9(17.3)$ & 0.906 \\
Clinical characteristics & & & \\
Age, years & $59.27 \pm 6.92$ & $59.83 \pm 6.69$ & 0.675 \\
Basic SCr, $\mu$ mol/l & $217.5 \pm 68.1$ & $215.8 \pm 74.0$ & 0.903 \\
Highest SCr, $\mu$ mol/l & $690.0 \pm 94.0$ & $707.7 \pm 85.6$ & 0.312 \\
Organ failure, $\mathrm{n}$ & $1.76 \pm 0.69$ & $1.83 \pm 0.62$ & 0.620 \\
Duration of oliguria, days & $6.29 \pm 1.26$ & $6.69 \pm 1.41$ & 0.122 \\
\hline
\end{tabular}

$n=$ Number of organs experiencing failure.

${ }^{1}$ Figures represent the number of patients with the percentage in parentheses.

\begin{tabular}{lccccc}
\hline & \multicolumn{2}{l}{ CRRT } & & & \multicolumn{2}{l}{ SLEDD-f } \\
\cline { 2 - 3 } \cline { 5 - 6 } & pretreatment & posttreatment & & pretreatment & posttreatment \\
\hline APACHE-II score & $20.5 \pm 4.57$ & $13.8 \pm 3.5$ & & $20.8 \pm 3.4$ & $14.6 \pm 2.7$ \\
SOFA score & $13.3 \pm 2.8$ & $7.29 \pm 1.51$ & & $13.5 \pm 2.0$ & $7.35 \pm 1.22$ \\
BUN, $\mathrm{mmol} / \mathrm{l}$ & $23.69 \pm 2.7$ & $10.57 \pm 2.28$ & & $23.9 \pm 2.2$ & $10.87 \pm 1.76$ \\
$\mathrm{SCr}, \mu \mathrm{mol} / \mathrm{l}$ & $690.0 \pm 94.0$ & $198.3 \pm 37.3$ & & $707.7 \pm 85.6$ & $206.8 \pm 29.4$ \\
$\mathrm{CRP}, \mathrm{mg} / \mathrm{ml}$ & $19.8 \pm 4.3$ & $5.67 \pm 1.32$ & & $19.9 \pm 4.7$ & $5.51 \pm 1.78$ \\
$\mathrm{Hs}-\mathrm{CRP}, \mathrm{mg} / 1$ & $8.5 \pm 1.2$ & $2.35 \pm 0.51$ & & $8.1 \pm 1.5$ & $2.33 \pm 0.34$ \\
$\mathrm{PCT}, \mathrm{ng} / \mathrm{ml}$ & $1.48 \pm 0.73$ & $0.32 \pm 0.08$ & & $1.61 \pm 0.70$ & $0.33 \pm 0.10$ \\
WBC,$\times 10^{9} / 1$ & $12.5 \pm 2.9$ & $6.62 \pm 1.35$ & & $12.2 \pm 2.8$ & $6.93 \pm 1.50$ \\
\hline
\end{tabular}

$\mathrm{BUN}=$ Blood urea nitrogen; Hs-CRP = high-sensitivity CRP; $\mathrm{WBC}=$ white blood cell count.
Table 2. Comparison of clinical characteristics and inflammation indexes after RRT
SLEDD-f and 15,230 $\pm 1,460 \mathrm{U} /$ day for patients treated with CRRT; this difference was also statistically significant $(p=0.000)$. The cost of hemopurification for patients treated with SLEDD-f was USD 1,950 \pm 230 , significantly less than the USD $4,210 \pm 820$ for patients treated with CRRT ( $p<0.01$ ). Furthermore, the total expense of hospitalization for patients treated with SLEDD- $f$ was lower than that for patients treated with CRRT (USD 2,900 \pm 390 vs. USD 5,710 \pm 930 , respectively, as shown in fig. 1).

\section{Discussion}

In this study, the mortality and renal recovery rates were similar in the patients treated with CRRT and SLEDD-f, thereby indicating that these treatment modal- 
ities have the same effect on AKI/DN patients. The high survival rate was possibly due to timeous hemopurification treatment. Meanwhile, SLEDD-f offered other advantages including a lower heparin dosage, shorter therapy time and lower hospitalization costs than for CRRT. Episodes of technical failure (with the machines) or of compromised care were not observed with either treatment modality. SLEDD or SLEDD-f consists of an adaptation of conventional IHD or hemodiafiltration, aimed to be low efficient. This low efficiency was achieved by decreasing blood and dialysate pump velocity and extending treatment time to reduce hourly fluid removal. In 1999, Schlaeper et al. [22] treated critically ill patients requiring RRT by slow continuous dialysis defined by the following parameters: a blood flow of $100-200 \mathrm{ml} / \mathrm{min}$, a dialysate flow of $100-300 \mathrm{ml} / \mathrm{min}$, the use of a modified hemodialysis machine with controlled UF and online production of bicarbonate-based dialysate, and continuous or extended daily treatment of 8-24 h. This treatment was successful and since then, the role of SLEDD in AKI has gained more and more attention $[9,12,15]$. In addition, SLEDD has shown good effects in the treatment of critically ill patients or multiple-organ dysfunction syndrome [10, 23, $24]$ and acute poisoning $[25,26]$.

Likewise, SLEDD-f has emerged as a predictor for achieving the UF volume initially desired. Moreover, the dialytic efficacy of SLEDD-f reported in the literature is at least comparable to that of continuous therapies using high-dialysate and substitution fluids [27]. Meanwhile, SLEDD-f can alleviate the inflammatory response of AKI/ DN patients as well as CRRT can, demonstrated by how serum CRP and PCT both declined after treatment.

Our study also indicated that, compared to continuous therapies, SLEDD-f requires less anticoagulation agent due to a shorter dialytic period. Moreover, the application of SLEDD-f is a protector factor of coagulation. In this study, less hemorrhage occurred during SLEDD-f due to the shorter RRT time and less total heparin being applied; this could have some beneficial impact on patients at a high risk for hemorrhagic complications. The reduced treatment time of SLEDD-f means that at least 2 patients can be treated with the same dialysis monitor within $24 \mathrm{~h}$. Furthermore, unlike with CRRT, it is easy to decide when to switch from SLEDD-f to a conventional IHD.

SLEDD-f is also less costly and involves a smaller workload. Unlike CRRT, the SLEDD-f technique is easy for nurses to learn and use and it requires less monitoring and intervention. Thus, it is more suitable for economically undeveloped areas or areas with a scarcity of health resources. When compared to IHD, to the best of our knowledge, the availability of nursing staff was the only limiting factor for providing SLEDD-f treatment.

\section{Conclusion}

Our data suggest that SLEDD-f treatment has adequate small solute clearance, significant large solute clearance and excellent clinical, metabolic and economic outcomes in AKI/DN patients. In addition, this treatment can be delivered autonomously in the ICU and other nonnephrology departments by in-house nursing personnel in a similar manner to CRRT. This is logistically attractive to many units, especially in primary hospitals.

\section{Disclosure Statement}

The authors report no conflicts of interest. The authors alone are responsible for the content and writing of the paper.

\section{References}

-1 Abdul-Rasoul M, AlOtaibi F, Abdulla A, et al: Quality of life of children and adolescents with type 1 diabetes in Kuwait. Med Princ Pract 2013;22:379-384.

$\checkmark 2$ Farzaneh S, Mohaddeseh A, Nasser S, et al: Female sexual dysfunction in type 2 diabetes: a case control study. Med Princ Pract 2012;21: 554-559.

$\checkmark 3$ Ahmadi H, Karimi A, Davoodi S, et al: Determinant factors of renal failure after coronary artery bypass grafting with on-pump technique. Med Princ Pract 2009;18:300-304.
4 Yap SC, Lee HT: Acute kidney injury and extrarenal organ dysfunction: new concepts and experimental evidence. Anesthesiology 2012; 116:1139-1148.

$>5$ Uchino S, Bellomo R, Goldsmith D, et al: An assessment of the RIFLE criteria for acute renal failure in hospitalized patients. Crit Care Med 2006;34:1913-1917.

6 Bellomo R, Ronco C, Kellum JA, et al: Acute renal failure - definition, outcome measures, animal models, fluid therapy and information technology needs: the Second International Consensus Conference of the Acute Dialysis Quality Initiative (ADQI) Group. Crit Care 2004;8:R204-R212.
7 Kes P, Basic JN: Acute kidney injury in the intensive care unit. Bosn J Basic Med Sci 2010; 10(suppl 1):S8-S12.

-8 Susantitaphong P, Tiranathanagul K, Srisawat N, et al: Efficacy of separated system continuous venovenous hemofiltration in critical acute kidney injury. Ther Apher Dial 2011;15:475-480.

9 Kumar VA, Craig M, Depner TA, et al: Extended daily dialysis: a new approach to renal replacement for acute renal failure in the intensive care unit. Am J Kidney Dis 2000;36: 294-300.
Sustained Low-Efficiency Daily

Diafiltration in DN with AKI
Med Princ Pract 2014;23:119-124

DOI: $10.1159 / 000358172$ 
10 Marshall MR, Ma T, Galler D, et al: Sustained low-efficiency daily diafiltration (SLEDD-f) for critically ill patients requiring renal replacement therapy: towards an adequate therapy. Nephrol Dial Transplant 2004;19:877884.

11 Marshall MR, Golper TA, Shaver MJ, et al: Urea kinetics during sustained low-efficiency dialysis in critically ill patients requiring renal replacement therapy. Am J Kidney Dis 2002; 39:556-570.

12 Kumar VA, Yeun JY, Depner TA, et al: Extended daily dialysis vs. continuous hemodialysis for ICU patients with acute renal failure: a two-year single center report. Int J Artif Organs 2004;27:371-379.

13 Tolwani AJ, Wheeler TS, Wille KM: Sustained low-efficiency dialysis. Contrib Nephrol 2007; 156:320-324.

14 Baldwin I, Bellomo R, Naka T, et al: A pilot randomized controlled comparison of extended daily dialysis with filtration and continuous veno-venous hemofiltration: fluid removal and hemodynamics. Int J Artif Organs 2007;30:1083-1089.
15 Holt BG, White JJ, Kuthiala A, et al: Sustained low-efficiency daily dialysis with hemofiltration for acute kidney injury in the presence of sepsis. Clin Nephrol 2008;69:40-46.

16 Joannidis M: Good-bye CRRT, here comes SLED?... not so fast! Crit Care 2012;16:167.

17 Mogensen CE, Christensen CK, Vittinghus E: The stages in diabetic renal disease. With emphasis on the stage of incipient diabetic nephropathy. Diabetes 1983;32(suppl 2):64-78.

18 Ronco C, Levin A, Warnock DG, et al: Improving outcomes from acute kidney injury (AKI): report on an initiative. Int J Artif Organs 2007;30:373-376.

19 Knaus WA, Draper EA, Wagner DP, et al: APACHE II: a severity of disease classification system. Crit Care Med 1985;13:818-829.

20 Vincent JL, Moreno R, Takala J, et al: The SOFA (Sepsis-Related Organ Failure Assessment) score to describe organ dysfunction/ failure. On behalf of the Working Group on Sepsis-Related Problems of the European Society of Intensive Care Medicine. Intensive Care Med 1996;22:707-710.

21 Bellomo R, Ronco C: Continuous renal replacement therapy in the intensive care unit. Intensive Care Med 1999;25:781-789.

22 Schlaeper C, Amerling R, Manns M, et al: High clearance continuous renal replacement therapy with a modified dialysis machine. Kidney Int Suppl 1999;72:S20-S23.
23 Marshall MR, Golper TA, Shaver MJ, et al: Sustained low-efficiency dialysis for critically ill patients requiring renal replacement therapy. Kidney Int 2001;60:777-785.

24 Cheng J, Hu S, Lu H, et al: Comparison of the therapeutic effectiveness of sustained low-efficiency dialysis (SLED) with continuous blood purification (CBP) in critically ill patients. Cell Biochem Biophys 2013;67:923927.

25 Wu CL, Chiu PF, Yang Y, et al: Sustained lowefficiency daily diafiltration with hemoperfusion as a therapy for severe star fruit intoxication: a report of two cases. Ren Fail 2011;33: 837-841.

26 Khan E, Huggan P, Celi L, et al: Sustained low-efficiency dialysis with filtration (SLEDD-f) in the management of acute sodium valproate intoxication. Hemodial Int 2008;12:211-214.

27 Fieghen HE, Friedrich JO, Burns KE, et al: The hemodynamic tolerability and feasibility of sustained low efficiency dialysis in the management of critically ill patients with acute kidney injury. BMC Nephrol 2010;11: 32. 